\title{
Frontal Lobe Epilepsy: A Great Masquerader in Psychiatry
}

\section{Ahmed Naguy ${ }^{1 *}$ and Ali Al-Tajali ${ }^{2}$}

${ }^{1}$ Child and Adolescent Psychiatrist, Kuwait Centre for Mental Health, Kuwait

${ }^{2}$ General Adult Psychiatrist, Neuromodulation \& Psych Rehab, Kuwait Centre for Mental Health, Kuwait

Keywords: Frontal lobe epilepsy; Pavor nocturnus; Nocturnal enuresis-imipramine

\section{To the Editor}

Frontal lobe epilepsy (FLE) is characterized by frequent seizures in stage 2 sleep, short duration, minimal postictal confusion, rapid secondary generalization, prominent motor manifestations, complex gestural automatisms at onset, frequent falling during seizure, and, frequent episodes of status epilepticus [1,2].

Frontal lobe seizures may produce unusual symptoms that can appear to be related to a psychiatric problem or a sleep disorder [3-5] (Table 1). Here, we are reporting a case of FLE that masqueraded as pavor nocturnus and nocturnal enuresis (NE). This had very serious implications.

A 7-year-old Egyptian male youngster was escorted by his mother. She reported her son lately experienced discrete sudden episodes of awakening from sleep in the middle of night screaming, pallid, sweating, inconsolable, and, totally amnestic to the event the next morning. Occasionally, there is accompanying bedwetting. He is the eldest of 3 siblings, product of monogamous, consanguineous marriage, elective C.S., and, neurotypically developing. No family history of neuropsychiatric history. No medical history of note. He was then diagnosed as pavor nocturnus with nocturnal enuresis. As these 'spells' began to negatively impact his performance at school as he usually feels fatigued next morning, he was prescribed imipramine $25 \mathrm{mg} / \mathrm{d}$ to help both parasomnias. Over 2 weeks, dose uptitrated to $75 \mathrm{mg} / \mathrm{d}$. At this point, kid had status epilepticus and was hospitalized. Ictal EEG (transverse montage) revealed FLE. And NE was in fact urinary incontinence accompanying seizure activity. Imipramine ceased, and it seems it had precipitated status, as TCAs are notorious to decrease the seizure threshold [6]. He was maintained on carbamazepine with good control of seizures. No more sleep terrors or enuresis.

Given the complex and atypical semiology of FLE, we opine that EEG should be an integral part of workup for any complex sleep phenomena before embarking on medications [7-8] (Table 2). This might preclude the oftentimes catastrophic sequelae of missing a great masquerader as FLE.

\begin{tabular}{|c|c|}
\hline FLE & Parasomnia \\
\hline "stereotypic & -variable \\
\hline -more frequent and clustering & - less frequent \\
\hline -commonly, shortly after falling asleep & within 2 hs of sleep onset \\
\hline -prominent tonic component & -ill-defined \\
\hline -brief, less than 2 mins & -prolonged \\
\hline -persists into adulthood & - limited to childhood \\
\hline
\end{tabular}

Table 1: Differentiating FLE vs. Parasomnia.

\section{-History}

-FLEP: Frontal Lobe Epilepsy and Parasomnia Scale

-Video-EEG-Polysomnography

Table 2: Workup to differentiate FLE vs. parasomnias.

\section{Disclosures}

Authors declare no conflict of interest, or financial affiliations with pharmaceutical companies, or, industry-sponsored research.

\section{References}

1. Salanova V, Morris HH, Van Ness P, Kotagal P, et al. (1995) Frontal lobe seizures: electroclinical syndromes. Epilepsia 36: 16-24.

2. Bonelli SB, Baumgartner C (2002) Frontal lobe epilepsy-clinical seizure semiology. Wein Klin Wochenschr 114: 334-337.

3. Bourion-Bedes S, Hingray C, Faust H, Vignal JP, Vespignani H, et al. (2014) Pitfalls in the diagnosis of new-onset frontal lobe seizures. Epilepsy \& Behavior Case Reports 2: 1-3.

4. Butler C, Zeman AZJ (2005) Neurological syndromes which can be mistaken for psychiatric conditions. J Neurol Neurosurg Psychiatry 76: i31-i38.

5. Boursoulian LJ, Schenck CH, Mahowald MW, Lagrange AH (2012) Differentiating Parasomnias from Nocturnal Seizures. J Clin Sleep Med 8: 108112.

6. Preskorn SH, Fast GA (1992) Tricyclic antidepressant-induced seizures and plasma drug concentration. J Clin Psychiatry 53: 160-162.

7. Smith SJM (2005) EEG in neurological conditions other than epilepsy: when does it help, what does it add? J Neurol Neurosurg Psychiatry 76: ii8-ii12.

8. Derry C (2012) Nocturnal Frontal Lobe Epilepsy vs. Parasomnia. Curr Treat Options Neurol 14: 451-463.

*Corresponding author: Ahmed Naguy, Child and Adolescent Psychiatrist, Kuwait Centre for Mental Health (KCMH), Shwaikh, State of Kuwait, Tel: +965/65541937; E-mail: ahmednagy@hotmail.co.uk

Received June 03, 2015; Accepted August 02, 2015; Published August 10, 2015

Citation: Naguy A, Al-Tajali A (2015) Frontal Lobe Epilepsy: The Great Masquerader in Psychiatry. Brain Disord Ther 4:176. doi:10.4172/2168-975X.1000176

Copyright: @ 2015 Naguy A, et al. This is an open-access article distributed under the terms of the Creative Commons Attribution License, which permits unrestricted use, distribution, and reproduction in any medium, provided the original author and source are credited. 